\title{
The molecular neurobiology of depression
}

\author{
Vaishnav Krishnan ${ }^{1,2}$ and Eric J. Nestler ${ }^{1,2,3}$ \\ ${ }^{1}$ Department of Psychiatry, University of Texas Southwestern Medical Center, Dallas, Texas 75390, \\ USA \\ 2 Department of Neuroscience, University of Texas Southwestern Medical Center, Dallas, Texas \\ 75390, USA \\ ${ }^{3}$ Fishberg Department of Neuroscience, Mount Sinai School of Medicine, New York, New York \\ 10029, USA
}

\section{Abstract}

Unravelling the pathophysiology of depression is a unique challenge. Not only are depressive syndromes heterogeneous and their aetiologies diverse, but symptoms such as guilt and suicidality are impossible to reproduce in animal models. Nevertheless, other symptoms have been accurately modelled, and these, together with clinical data, are providing insight into the neurobiology of depression. Recent studies combining behavioural, molecular and electrophysiological techniques reveal that certain aspects of depression result from maladaptive stress-induced neuroplastic changes in specific neural circuits. They also show that understanding the mechanisms of resilience to stress offers a crucial new dimension for the development of fundamentally novel antidepressant treatments.

\begin{abstract}
About one in six individuals in the United States will succumb to clinical depression during their lifetime ${ }^{1}$. Core symptoms include depressed mood, anhedonia (reduced ability to experience pleasure from natural rewards), irritability, difficulties in concentrating, and abnormalities in appetite and sleep ('neurovegetative symptoms') ${ }^{2}$. In addition to mortality associated with suicide, depressed patients are more likely to develop coronary artery disease and type 2 diabetes $^{3}$. Depression also complicates the prognosis of a host of other chronic medical conditions $\mathrm{s}^{4,5}$. The chronic, festering nature of depression contributes substantially to the global burden of disease and disability.

Despite the prevalence of depression and its considerable impact, knowledge about its pathophysiology is rudimentary compared with knowledge of other common chronic and potentially fatal multifactorial conditions, such as type 2 diabetes (Table 1). There are several explanations for this discrepancy. First and foremost, observing pathological changes within the brain remains markedly more difficult than for all other organs. Available techniques to document the aberrant function of brain circuits depend on either post-mortem studies, which have numerous limitations, or neuroimaging techniques, which rely on detecting changes in neuronal activity by using indirect markers of activation ${ }^{6}$. Although these approaches have provided important insights into candidate brain regions, simple increases or decreases in regional brain activity are probably insufficient to explain the complex array of symptoms caused by depression. Several animal models have also informed knowledge of the neural
\end{abstract}

Correspondence should be addressed to E.J.N. (E-mail: eric.nestler@mssm.edu).

Author Information Reprints and permissions information is available at www.nature.com/reprints

The authors declare competing financial interests: details accompany the full-text HTML version of the paper at www.nature.com/nature. 
circuitry of depression, but there are important challenges to how information gained from these models should be interpreted (Box 1).

Second, most depression occurs idiopathically, and the limited understanding of its aetiology is reflected as a list of risk factors, such as stressful life events, endocrine abnormalities (hypothyroidism and hypercortisolism), cancers (such as pancreatic adenocarcinoma and breast tumours) and side effects of drugs (for example, isotretinoin for acne, and interferon$\alpha$ for hepatitis $C$ ), among many others ${ }^{2,4,7}$. Genetic association studies have not uncovered strong and consistent genetic risk modifiers ${ }^{8}$, perhaps because of the sheer heterogeneity of depressive syndromes ${ }^{2,9}$. Thus, genuine 'depression genes', which can be used to generate disease models in mice (for example, those for Rett syndrome or familial Alzheimer's disease), have not yet been identified. Genetic predispositions are thought to interact with environmental risk factors, such as stressful life events, which can initiate depressive episodes in some patients ${ }^{10}$. Still, the tendency to live in high-stress environments might also be partly heritable (as is the case for 'risk or sensation seekers') ${ }^{11}$, emphasizing the strong genetic contribution to even 'environmentally precipitated' depressive episodes.

The official diagnosis of depression is subjective and rests on the documentation of a certain number of symptoms that significantly impair functioning for a certain duration ${ }^{2}$. These diagnostic criteria overlap with other conditions such as anxiety disorders, which have substantial co-morbidity with depression ${ }^{12,13}$. Therefore, two 'depressed' patients might have only one symptom in common ${ }^{7}$, and a manic episode in one patient - even later in life switches the diagnosis to bipolar disorder, which is presumably a distinct pathophysiological entity. This symptom-based diagnostic approach poses obvious obstacles to the interpretation of genome-wide association studies, as well as neuroimaging and post-mortem investigations.

In this Review, we summarize the current state of knowledge of the neural and molecular mechanisms of depression. We focus on key leading hypotheses in the field, and examine their strengths and weaknesses critically in the light of recent preclinical and translational studies. We also highlight new insights that promise to extend the understanding of depression and improve its treatment.

\section{Neural circuitry of depression}

Several brain regions and circuits regulate emotion, reward and executive function, and dysfunctional changes within these highly interconnected 'limbic' regions have been implicated in depression and antidepressant action ${ }^{14}$ (Fig. 1). A large body of post-mortem ${ }^{7}$, 15 and neuroimaging ${ }^{7,16}$ studies of depressed patients have reported reductions in grey-matter volume and glial density in the prefrontal cortex and the hippocampus, regions thought to mediate the cognitive aspects of depression, such as feelings of worthlessness and guilt. However, the published findings are not consistent and are often complicated by co-morbid diagnoses and medication history, and there has been limited success in demonstrating any clear cause-effect relationships of these pathological changes.

In contrast to structural studies, experiments assessing brain function, such as functional magnetic resonance imaging (fMRI) or positron-emission tomography (PET), show that activity within the amygdala and subgenual cingulate cortex $(\mathrm{Cg} 25$, a subregion of prefrontal cortex) is strongly correlated with dysphoric emotions: indices of neuronal activity within these regions are increased by transient sadness in healthy volunteers and are chronically increased in depressed individuals, reverting to normal levels with successful treatment ${ }^{7,13}$. Inspired by these findings, it was shown that deep brain stimulation applied to the white matter tracts surrounding $\mathrm{Cg} 25$ produced a sustained remission of depressive symptoms in a small cohort of treatment-resistant patients (patients who failed to respond to several standard treatments) 17 . Deep brain stimulation, achieved through the stereotactic surgical placement of stimulating 
electrodes, has also provided an acute ameliorative effect on clinical ratings when applied to the nucleus accumbens (NAc) ${ }^{18}$, a striatal subregion that is important for reward and for hedonic deficits in depression ${ }^{19}$.

These forebrain networks are significantly modulated by monoamine projections from midbrain and brainstem nuclei (dopamine from the ventral tegmental area (VTA), serotonin from the dorsal raphe located in the periaqueductal grey area, and noradrenaline from the locus coeruleus). In addition to controlling alertness and awareness, these neurotransmitters modulate the salience of emotional stimuli. More recent studies have investigated the role of specific hypothalamic nuclei in mediating the neurovegetative signs of depression. However, we add a note of caution: although depressive symptoms are probably mediated by dysfunction in a diffuse series of neural networks, the field has often used a simplistic 'localization of function' approach to examine limbic substrates (for example, amygdala $\approx$ 'fear and anxiety', $\mathrm{NAc} \approx$ 'reward'). Such artificial distinctions are of limited heuristic value and reflect limitations in the ability of current technologies to understand systems-level dysfunction.

\section{The role of monoamines}

The 'monoamine hypothesis' of depression, which posits that depression is caused by decreased monoamine function in the brain, originated from early clinical observations ${ }^{14,20}$. Two structurally unrelated compounds developed for non-psychiatric conditions, namely iproniazid and imipramine, had potent antidepressant effects in humans and were later shown to enhance central serotonin or noradrenaline transmission. Reserpine, an old antihypertensive agent that depletes monoamine stores, produced depressive symptoms in a subset of patients. Today's antidepressant agents offer a better therapeutic index and lower rates of side effects for most patients, but they are still designed to increase monoamine transmission acutely ${ }^{14}$, either by inhibiting neuronal reuptake (for example, selective serotonin reuptake inhibitors (SSRIs) such as fluoxetine) or by inhibiting degradation (for example, monoamine oxidase inhibitors such as tranylcypromine). Although these monoamine-based agents are potent antidepressants $^{21}$, and alterations in central monoamine function might contribute marginally to genetic vulnerability ${ }^{8,22}$, the cause of depression is far from being a simple deficiency of central monoamines. Monoamine oxidase inhibitors and SSRIs produce immediate increases in monoamine transmission, whereas their mood-enhancing properties require weeks of treatment. Conversely, experimental depletion of monoamines can produce a mild reduction in mood in unmedicated depressed patients, but such manipulations do not alter mood in healthy controls $^{23}$. Moreover, studies with rodent stress models have shown that enhancements in dopamine and noradrenaline transmission can have maladaptive roles in stress-related disorders by strengthening memories of aversive life events ${ }^{24,25}$.

It is now thought that acute increases in the amount of synaptic monoamines induced by antidepressants produce secondary neuroplastic changes that are on a longer timescale and involve transcriptional and translational changes that mediate molecular and cellular plasticity 2,20 . As one example, the serotonin $5-\mathrm{HT}_{1 \mathrm{~B}}$ receptor interacts with a calcium-binding protein named p11, which was upregulated in cerebral cortex on chronic treatment with SSRIs and was also found to be downregulated in post-mortem cingulate cortex samples from depressed individuals ${ }^{26}$. The brain-specific transgenic overexpression of $\mathrm{p} 11$ produced an antidepressant phenotype, implicating this SSRI-mediated upregulation of p11 as an important mechanism downstream of serotonin receptor activation. Chronically administered antidepressants have also been shown to upregulate the transcription factor CREB (cyclic-AMP-response-elementbinding protein), which is downstream of several serotonin and other stimulatory G-proteincoupled receptors, in the hippocampus; this effect has been validated in human post-mortem tissue and directly linked to antidepressant-like responses in animal models ${ }^{2,20}$. By contrast, stress activation of CREB in NAc triggers depression-like responses, which underscores crucial 
region-specific actions of neurotransmitters and their downstream effectors that have not been incorporated into simplistic deficiency models ${ }^{19}$.

Monoamine-based antidepressants remain the first line of therapy for depression, but their long therapeutic delays and low (about 30\%) remission rates ${ }^{21}$ have encouraged the search for more effective agents ${ }^{14,27}$. The serotonin receptors involved in the action of SSRIs remain unknown, although selective agonists of the serotonin $5-\mathrm{HT}_{4}$ receptor produce rapid antidepressant effects in rodents (three to four days) ${ }^{28}$. Experiments on mice deficient in P-glycoprotein, a molecule in the blood-brain barrier that transports numerous drugs back into the bloodstream, have shown that several antidepressant agents, including the SSRI citalopram, are substrates for P-glycoprotein. Human polymorphisms in the gene encoding P-glycoprotein significantly alter antidepressant efficacy in depressed individuals ${ }^{29}$, suggesting the value of such a pharmacogenetic approach when selecting antidepressant agents ${ }^{30}$.

\section{Box 1}

\section{Preclinical models}

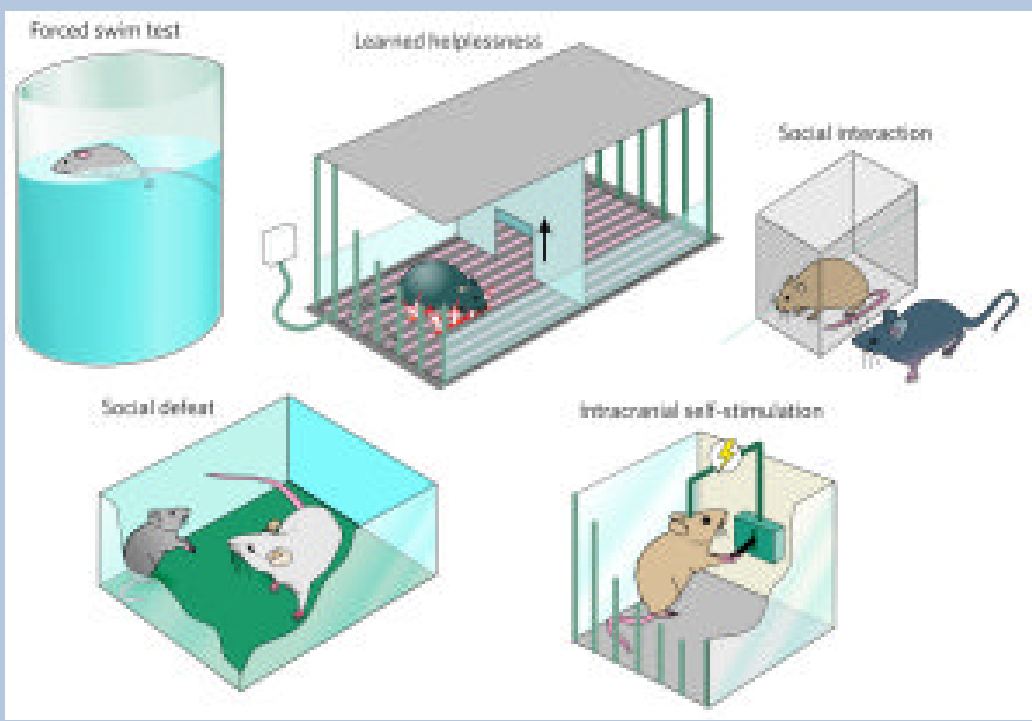

Animal models of depression are evaluated for their aetiological validity: to be valid, depression-like behaviours need to be caused by the same aetiologies that trigger human depression. This is a challenging requirement, given the absence of definitive aetiologies for human depression. Current models gauge an animal's 'depression-related' responses to acute or chronic inescapable stress. These include the forced-swim test ${ }^{35,53,73}$, which quantifies immobility in a water bath (proposed to be analogous to 'behavioural despair' observed in depression; see box figure, left). Other assays include measuring social interaction (decreases in which may model social withdrawal of depression-related conditions) $)^{25,39,79,95}$, the learned-helplessness test (which measures the development of passive responses to inescapable foot shock) ${ }^{84}$, and intracranial self-stimulation, an operant measure of the effort that an animal expends to stimulate brain reward circuits electrically ${ }^{14,23}$. Several of these show pharmacological validity - that is, they are sensitive to acutely administered known antidepressant compounds ${ }^{14,99}$ - which permits the rapid screening of potentially new therapeutic agents. However, because more than half of all depressed individuals do not respond fully to available antidepressants ${ }^{21}$, the requirement for pharmacological validity is a circular argument that deserves reconsideration. Models that use an acute stress (for example, forced swimming) are better thought of as 'tests' of coping behaviour, and are limited in their ability to recapitulate a long-lived 
multidimensional syndrome such as depression. Efforts to create the latter are almost entirely limited to chronic stress models such as chronic social defeat or chronic mild stress, which are more technically challenging but show unique sensitivity to chronic and not acute antidepressant administration, comparable to the therapeutic delay of 4-6 weeks that is required for all available antidepressant drugs to treat depression in humans ${ }^{2}$. Animal models also have face validity, in which certain behavioural changes brought about by stress or genetic manipulation superficially resemble depressive symptoms. For instance, an animal's decreased sucrose intake after chronic stress is thought to model anhedonia ${ }^{25}$.

These tests have been applied to the study of the molecular neurobiology of depression in three main ways. The most popular approach documents neuroplastic changes in brain regions after chronic stress and has revealed a role for structural, transcriptional and epigenetic changes in several brain regions (for example, those shown in Figs 1-3). These models can also be used to examine the behavioural effects of region-specific genetic manipulation, achieved through targeted genetic mutations in mice or virus-mediated gene transfer. The selective breeding of extreme populations within outbred rodents has also been used to generate stress-vulnerable or stress-resistant inbred strains (not shown) ${ }^{17}$. This approach is particularly valuable for quantitative trait locus (QTL) analyses, as well as for dissecting epigenetic contributions to responsiveness to stress ${ }^{25}$. These behavioural assays can also be used to study biological mechanisms that underlie phenotypic variations in stress responses. For example, susceptibility to social defeat is mediated by increases in the electrical activity of dopamine-producing neurons in the ventral tegmental area (VTA) ${ }^{25}$, whereas resistance to learned helplessness is mediated by induction of the gene encoding the transcription factor $\triangle F O S B$ in the periaqueductal grey (PAG) area ${ }^{84}$ (see box figure, right). In these ways, preclinical models of depression have provided important insights into the pathophysiology of depression.

\section{Neurotrophins and neurogenesis}

Volumetric decreases observed in the hippocampus and other forebrain regions in subsets of depressed patients have supported a popular hypothesis for depression involving decrements in neurotrophic factors - neurodevelopmentally expressed growth factors that also regulate plasticity within adult brain ${ }^{31,32}$. These studies have focused largely on the role of brainderived neurotrophic factor (BDNF), which is expressed abundantly in adult limbic structures. Support for this 'BDNF hypothesis' has come from a large preclinical literature showing that several forms of stress reduce BDNF-mediated signalling in the hippocampus, whereas chronic treatment with antidepressants increases BDNF-mediated signalling 2,31 . Similar changes have been observed in the post-mortem hippocampus of humans with depression ${ }^{33}$, as well as in the concentrations of serum BDNF, the source of which remains controversial ${ }^{31}$.

More causal evidence for the antidepressant action of BDNF has come from experiments in rodents in which antidepressant effects were observed on direct infusion of BDNF into the hippocampus ${ }^{34}$ and were blocked on the conditional or inducible knockout of the gene encoding BDNF from forebrain regions ${ }^{32,35}$. However, more recent findings have necessitated a revision of this hypothesis. First, a substantial number of preclinical studies either have failed to show these patterns of changes induced by stress and by antidepressants, or have shown the opposite effects ${ }^{36,37}$. Second, male mice with conditional forebrain deletions of BDNF or its receptor do not show depression-like behaviour ${ }^{35,38}$. Third, in other regions - for example the VTA and NAc - BDNF exerts a potent pro-depressant effect: chronic stress increases the amount of BDNF within the NAc ${ }^{39}$, and the direct infusion of BDNF into the VTA-NAc increases depression-related behaviours ${ }^{25,40}$, whereas a selective knockout of the gene encoding BDNF from this circuit has antidepressant-like effects ${ }^{39}$. Finally, a single-nucleotide polymorphism (G196A; Val $663 \rightarrow$ Met 66) in the gene encoding BDNF, which significantly 
impairs the intracellular trafficking and activity-dependent release of $\mathrm{BDNF}^{41,42}$ and decreases hippocampal volume ${ }^{41,43}$, does not alter genetic vulnerability to depression ${ }^{8,44}$ (Fig. 2). In addition, recent studies suggest complex interactions between the BDNF G196A polymorphism, a polymorphism in the serotonin transporter gene, and stressful life events ${ }^{45-}$ 47. Taken together, these results suggest that the current formulation of the BDNF hypothesis is too simplistic; BDNF-mediated signalling is involved in neuroplastic responses to stress and antidepressants, but these effects are both region-specific ${ }^{19}$ and antidepressant-specific ${ }^{31}$ and function in the background of other potent genetic and environmental modifiers.

A marked cellular effect of several, but not all, antidepressant treatments is the induction of adult hippocampal neurogenesis - the process by which neural progenitors of the hippocampal subgranular zone (SGZ) divide mitotically to form new neurons that differentiate and integrate into the dentate gyrus ${ }^{20,48}$. Blockade of hippocampal neurogenesis inhibits the therapeuticlike effects of most antidepressant treatments in rodent models ${ }^{48}$. Moreover, treatment with antidepressants, possibly through the actions of CREB or other transcriptional regulators ${ }^{2,20}$, increases the amounts of several growth factors in the hippocampus that influence neurogenesis. These include BDNF (which promotes neuronal survival ${ }^{49}$ ), as well as vascular endothelial growth factor (VEGF) and VGF, which themselves have antidepressant and proneurogenic properties in rodents ${ }^{50-52}$. The mechanisms by which new neurons might restore mood are largely unknown. Activity-dependent increases in neurogenesis might increase activity propagation through hippocampal subfields ${ }^{53}$ and allow hippocampal networks to adapt and learn new experiences ${ }^{54}$. Indeed, this raises the possibility that the presence of intact neurogenesis during stressful episodes mediates maladaptive learning and thus promotes depressive sequelae. Whereas several types of stress reduce SGZ cell proliferation, decreased neurogenesis does not itself produce depression ${ }^{48,55}$ : rodents in which hippocampal neurogenesis has been ablated (through either irradiation ${ }^{55,56}$ or genetic techniques ${ }^{57}$ ) do not show anxiety-related or depression-related behaviours.

Collectively, these studies highlight the weaknesses of attempts to generate a 'unified theory' of depression. Mechanisms that promote depressive symptoms in response to stress differ markedly between different neural circuits and can also be distinct from changes that underlie depression in the absence of external stress ('endogenous depression'). In addition, neuroplastic events that are required for antidepressant efficacy need not function through the reversal of stress-induced plasticity ${ }^{2}$, and might function through separate and parallel circuits.

\section{Neuroendocrine and neuroimmune interactions}

Early clinical studies identifying reproducible but small increases in serum glucocorticoid concentrations in depression ${ }^{58,59}$ fuelled significant interest in the role of a dysfunctional hypothalamic-pituitary-adrenal axis in the pathophysiology of depression. Physical or psychological stress increases serum glucocorticoid concentrations, and some depression-like symptoms can be produced in rodents by chronic administration of glucocorticoids ${ }^{60}$. Excess glucocorticoids, through the activation of glucocorticoid receptors, can reduce SGZ proliferation rates and produce atrophic changes in hippocampal subregions ${ }^{61}$. This could contribute to the hippocampal volume reductions seen in depression. Patients with Cushing's syndrome, who have extremely high concentrations of circulating cortisol, also show depressive features and atrophic changes in the hippocampus ${ }^{2,61}$. Several metabolic abnormalities that are often associated with depression, such as insulin resistance and abdominal obesity, can be at least partly explained by an increase in glucocorticoids ${ }^{4,62}$. Hypercortisolaemia in depression is manifested at several levels, including impaired glucocorticoid-receptor-mediated negative feedback ${ }^{62}$, adrenal hyper-responsiveness to circulating adreno-corticotropic hormone (ACTH) ${ }^{58}$ and hypersecretion of cortico tropinreleasing factor ${ }^{63}$, the hypothalamic activator of ACTH release from the pituitary ${ }^{2,64}$. In line 
with these findings, glucocorticoid and corticotropin-releasing factor receptor antagonists are currently being tested in clinical trials ${ }^{27}$.

More recent studies suggest that hypercortisolaemia is almost exclusively a feature of very severe depressive episodes, such as are observed in an in-patient setting ${ }^{65}$ or accompanied by psychotic symptoms (for example, hallucinations and delusions) ${ }^{2,9}$ in which glucocorticoid antagonists show some therapeutic efficacy ${ }^{66}$. By contrast, atypical depression, a subtype characterized by hyperphagia and hypersomnia, seems to be associated with hypocortisolaemia ${ }^{65,67}$, a phenomenon that is also observed in certain associated conditions such as fibromyalgia, chronic fatigue syndrome and post-traumatic stress disorder ${ }^{68}$. The origins of such distinct glucocorticoid profiles might reflect the evolutionary trade-off between the catabolic and immunosuppressant effects of glucocorticoids: whereas high serum concentrations of glucocorticoids promote the mobilization of energy resources during stressful experiences, low glucocorticoid states allow an unobstructed immune system to combat infection or physical injury sustained during adverse encounters in the wild ${ }^{59}$.

Cytokines, which are humoral mediators of innate and adaptive immunity, are also important modulators of mood. Cytokine receptors within the central nervous system are activated by both peripherally and centrally synthesized cytokines ${ }^{69}$. Low doses of lipopolysaccharide or interleukin 1 (IL-1) produce 'sickness behaviour' in rodents (consisting of social withdrawal and decreased exploratory and sexual behaviour), brought about by the release of proinflammatory cytokines such as interferon- $\alpha$, tumour necrosis factor- $\alpha$ (TNF- $\alpha$ ), IL- 6 and IL-1 $\beta$, which activate the hypothalamic-pituitary-adrenal axis and central monoamine systems ${ }^{70}$. Roughly $30 \%$ of individuals treated with recombinant interferons develop depression as a side effect of treatment ${ }^{71}$. Clinical studies examining depression-associated increases in serum cytokine concentrations have been largely inconsistent ${ }^{70}$. This suggests that immune activation is a signature of a small subset of depression cases, including those associated with autoimmune conditions such as rheumatoid arthritis, in which heightened system-wide inflammation can increase the risk of acute coronary events ${ }^{4}$ in addition to producing depressive mood changes.

Administration of cytokines such as interferon- $\alpha$ or IL- 6 to rodents does not cause consistent depression-like features ${ }^{70}$. Nevertheless, recent preclinical studies indicate that blocking proinflammatory cytokine-mediated signalling can produce antidepressant effects. Mice with targeted deletions of the gene encoding IL-6 (ref. ${ }^{72}$ ) or those encoding the TNF- $\alpha$ receptors ${ }^{73}$ show antidepressant-like behavioural phenotypes, and a centrally administered antagonist of the IL- $1 \beta$ receptor reversed the behavioural and antineurogenic effects of chronic stress $^{74}$. Future studies of the 'cytokine hypothesis' must focus on elucidating the largely unknown neural circuitry involved in the behavioural effects of cytokines, and must more precisely delineate the intercellular interactions involved between brain macrophages (microglia), glia and neurons within this circuitry.

\section{Epigenetic mechanisms}

Among the several methods by which experience can produce long-lasting changes in protein availability and function, there has been considerable recent interest in epigenetic modifications in the pathophysiology of depression and antidepressant action. These modifications (Fig. 3) encompass covalent changes to DNA (for example, DNA methylation) and post-translational modifications of histone $\mathrm{N}$-terminal tails (for example, acetylation and methylation), as well as non-transcriptional gene-silencing mechanisms (for example, microRNAs ${ }^{75}$. Given that these changes can be long-lasting, epigenetics has been invoked to explain several aspects of depression, including high discordance rates between monozygotic twins, individual differences among inbred rodents, the chronic relapsing nature of the illness, and 
the strikingly greater prevalence of depression in women ${ }^{11}$. In essence, epigenetic changes offer a mechanism by which environmental experiences can modify gene function in the absence of DNA sequence changes, and they might help to explain largely inconsistent genetic association studies of depression, for example by undermining the transcriptional impact of DNA sequence polymorphisms due to epigenetic modifications on those gene promoters ${ }^{11}$.

Although epigenetic changes have been implicated in numerous psychiatric conditions ${ }^{75}$, the field of depression research has focused on two main chromatin-modifying processes. The first is DNA methylation (of cytosine), which seems to be important in the influence of maternal behaviour on adult emotional processing. Adult offspring of rats born to mothers with low rates of maternal licking and grooming show increased anxiety and reduced expression of glucocorticoid receptors within the hippocampus compared with offspring of mothers with high rates of maternal behaviours. This reduced expression of glucocorticoid receptors is mediated by increased methylation of the glucocorticoid receptor gene promoter (effectively repressing gene expression). This long-lasting 'molecular scar ${ }^{75}$ is established within the first week of life and is effectively reversed by cross-fostering ${ }^{76}$. Interestingly, this increase in methylation was also reversed by the infusion of trichostatin A, a histone deacetylase (HDAC) inhibitor $^{77}$.

Histone acetylation, which is associated with transcriptional activation and decondensed chromatin, seems to be a key substrate for antidepressant action ${ }^{78}$. Increased histone acetylation at the $B d n f$ promoter in the hippocampus was shown to be required for the ability of chronically administered imipramine to reverse certain deleterious effects of social defeat ${ }^{79}$. Moreover, HDAC inhibitors show antidepressant-like effects in the social-defeat assay and other behavioural assays ${ }^{79,80}$, and efforts are underway to develop more potent agents that are designed to target specific HDACs, such as HDAC5, a class II HDAC ${ }^{75,79}$. The implications of these studies come with an important anatomical caveat: although inhibiting the actions of HDAC5 in the hippocampus seems to be therapeutically advantageous ${ }^{17,80}$, mice that are globally deficient in HDAC5 are more vulnerable to social defeat ${ }^{81}$. Similarly, although imipramine increases HDAC 5 expression in the hippocampus ${ }^{79}$, it significantly reduces HDAC5 expression within the $\mathrm{NAc}^{81}$, further emphasizing the regional specificity of stressrelated and antidepressant-related plasticity.

Current knowledge of the diversity of chromatin-modifying enzymes, and techniques to detect and quantify chromatin modifications genome-wide, is growing at an enormous pace. An important challenge in the clinical translation of these approaches will be to improve the technological ability to demonstrate causation by developing techniques to detect these modifications in vivo. Such techniques will allow researchers to examine, for the first time, region-specific chromatin measures associated with depression or antidepressant responses in humans.

\section{Resilience-related research}

Humans show a remarkable heterogeneity in their responses to stress and adversity: although a subset of depression cases can be causally attributed to stressful life events, these events in themselves raise only moderately the risk of developing depression ${ }^{10}$. In addition, reactive dysphoric states such as post-traumatic stress disorder only emerge in about 10-20\% of traumaexposed individuals ${ }^{82}$. Although a large body of research describes maladaptive neurobiological changes that occur after stressful exposures (such as decreased hippocampal neurogenesis and lower concentrations of BDNF, as discussed in the section 'Neurotrophins and neurogenesis'), relatively little attention has been devoted to understanding how most individuals adapt well — that is, are 'resilient' — in the face of adversity ${ }^{83}$. 
Animal models have recently been used to provide some neurobiological insight into these clinical observations. For example, by exploiting natural variations in the development of active escape in the learned-helplessness test, stress-induced upregulation of the transcription factor $\triangle F O S B$ (a stable, truncated protein product of the Fosb gene) in the midbrain periaqueductal grey nucleus was shown to promote a resilient phenotype. This effect was mediated through downregulating expression of substance $P$, a neuropeptide released during stress $^{84}$. A more recent report illustrated the role of mesolimbic dopamine-mediated signalling in emotional homeostatic mechanisms ${ }^{25}$. By adapting the social-defeat model ${ }^{39,79}$ of depression to examine the variations in response to chronic stress ${ }^{85}$, vulnerability to the development of social avoidance and other deleterious sequelae was shown to be mediated by the increased excitability of VTA dopamine neurons and their subsequent increased activitydependent release of BDNF onto NAc neurons. Resilient mice (which also show increased $\triangle$ FOSB concentrations ${ }^{84}$ ) escaped this increase in VTA neuronal excitability by upregulating voltage-gated potassium channels, which functions as a molecular compensation to restore normal excitability and maintain low levels of BDNF-mediated signalling in the NAc. Other putative mechanisms of resilience have come from clinical and preclinical investigations. One involves the release of neuropeptide $\mathrm{Y}$ from locus coeruleus nerve terminals onto amygdala neurons, which promotes resilient behaviour ${ }^{83,86}$. Interestingly, many of these studies report stable individual differences in stress responses among genetically inbred mice, strongly implicating non-genomic factors ${ }^{25,84,87}$. As these mice are housed under identical environmental conditions as well, the findings indicate the likely importance of epigenetic mechanisms during development, a possibility that now requires direct investigation.

Gene expression profiling of stress-vulnerable and stress-resilient mice revealed distinct transcriptional profiles in the VTA and NAc ${ }^{19}$, and similar results have been obtained in the hippocampus with related methods ${ }^{88}$. These findings suggest that resilient behaviour represents a distinct, active neurobiological process (not simply the absence of vulnerability) 25 . Accordingly, a comprehensive understanding of such molecular mechanisms of allostasis (ongoing efforts to maintain homeostasis) ${ }^{61}$ has the potential to be harnessed for the development of new therapeutic agents. In these ways, the identification of antivulnerability processes will be an important alternative approach to improving knowledge about the neurobiology of stress and the pathophysiology of depression.

\section{New insights}

Although the hypotheses described here remain active areas of research, recent findings have sparked interest in neurobiological systems that were previously unexplored in depression. A dramatic example is the observation that sub-anaesthetic doses of intravenously infused ketamine (a non-competitive NMDA ( $N$-methyl-D-aspartate) glutamate receptor antagonist and psychotomimetic) produce a rapid but transient antidepressant effect on individuals with treatment-resistant depression ${ }^{89}$. This effect suggests that depressive symptoms can be improved by altering the actions of glutamate, the major excitatory neurotransmitter in the brain. The antidepressant properties of ketamine have been recapitulated in animal tests of antidepressant action, such as the forced-swim test, in which the ability of ketamine to reduce immobility required intact signalling through AMPA receptors for glutamate ${ }^{90}$ and was associated with increased concentrations of hippocampal BDNF protein ${ }^{91}$. Despite the limited evidence for dysfunction in specific glutamate systems in depression, the clinical effects of ketamine have inspired new lines of preclinical research to explore the underlying neural circuitry and downstream signalling, as well as to identify previously unidentified NMDA receptor modulators that could be targeted to achieve better side-effect profiles ${ }^{92}$.

In the past few years, there has also been an increased interest in examining interactions between traditional mood substrates and pathways involved in the control of feeding and 
metabolism ${ }^{29}$. $\mathrm{MCH}$ (melanin-concentrating hormone)-containing neurons projecting from the lateral hypothalamus to several limbic regions including NAc provide an important orexigenic (pro-appetite) signal. Global decreases in MCH-mediated signalling ${ }^{93}$, as well as local $\mathrm{MCH}$ antagonism within the $\mathrm{NAc}^{94}$, produce antidepressant-like responses in several rodent models, generating tremendous interest in the antidepressant potential of selective $\mathrm{MCH}$ antagonists ${ }^{14}$, which might also curb the weight gain associated with a subset of depression ${ }^{19}$. In contrast to the pro-depressant actions of $\mathrm{MCH}$, other peptides such as orexin and ghrelin might have an antidepressant role, particularly during conditions of low caloric intake ${ }^{95}$. These and other studies illustrate the general theme that an animal's metabolic status greatly influences mood and motivation. Understanding the complex molecular interactions between peripheral metabolic signals (such as ghrelin ${ }^{95}$ and leptin ${ }^{96}$ ) and centrally released regulators of feeding and arousal (such as $\mathrm{MCH}$, orexin, neuropeptide $\mathrm{Y}^{83}$ and $\alpha$-melanocytestimulating hormone ${ }^{97}$ ) might provide new pathophysiological and therapeutic insights into mood disorders.

\section{Conclusion}

Knowledge of the pathophysiology of depression has evolved substantially: from Galen's speculations in antiquity about an excess of black bile ('melancholia') ${ }^{2,9}$ to theories focused on 'psychic pain' and 'chemical imbalances', and then to more current hypotheses that incorporate gene-environment interactions, endocrine, immunological and metabolic mediators, and cellular, molecular and epigenetic forms of plasticity. However, enormous gaps in the knowledge of depression and its treatment persist. Instead of being overwhelmed by the heterogen eity of the illness, researchers and clinicians must embrace the polysyndromic nature of depression and use a multidisciplinary approach to explore the neurobiological bases for depression's many subtypes. To improve the still-low remission rates ${ }^{21}$, it will be imperative to look beyond mono amine and neurotrophic mechanisms ${ }^{14}$ and expand knowledge about antidepressant pharmacogenetics. Researchers must better understand the biological basis for the efficacy of deep brain stimulation in depression, and must explore the therapeutic possibilities of viral-mediated gene delivery, which is being applied successfully to other neuropsychiatric disorders ${ }^{98}$. Finally, the field must harness the full potential of preclinical studies by continuing to develop improved animal models that incorporate the powerful array of molecular and anatomical tools available today, and must follow a systems approach to the study of depression that acknowledges the powerful bidirectional interactions between peripheral organs and the brain.

\section{Acknowledgments}

Work in E.J.N.'s laboratory was supported by grants from the National Institute of Mental Health.

\section{References}

1. Kessler RC, et al. Lifetime prevalence and age-of-onset distributions of DSM-IV disorders in the National Comorbidity Survey Replication. Arch Gen Psychiatry 2005;62:593-602. [PubMed: 15939837]

2. Nestler EJ, et al. Neurobiology of depression. Neuron 2002;34:13-25. [PubMed: 11931738]

3. Knol MJ, et al. Depression as a risk factor for the onset of type 2 diabetes mellitus. A meta-analysis. Diabetologia 2006;49:837-845. [PubMed: 16520921]

4. Evans DL, et al. Mood disorders in the medically ill: scientific review and recommendations. Biol Psychiatry 2005;58:175-189. [PubMed: 16084838]

5. Gildengers AG, et al. Medical burden in late-life bipolar and major depressive disorders. Am J Geriatr Psychiatry 2008;16:194-200. [PubMed: 18310550] 
6. Phelps EA, LeDoux JE. Contributions of the amygdala to emotion processing: from animal models to human behavior. Neuron 2005;48:175-187. [PubMed: 16242399]

7. Drevets WC. Neuroimaging and neuropathological studies of depression: implications for the cognitive-emotional features of mood disorders. Curr Opin Neurobiol 2001;11:240-249. [PubMed: 11301246]

8. Lopez-Leon S, et al. Meta-analyses of genetic studies on major depressive disorder. Mol Psychiatry 2007;13:772-785. [PubMed: 17938638]

9. Rush AJ. The varied clinical presentations of major depressive disorder. J Clin Psychiatry 2007;68 (Suppl 8):4-10. [PubMed: 17640152]

10. Kendler KS, Karkowski LM, Prescott CA. Causal relationship between stressful life events and the onset of major depression. Am J Psychiatry 1999;156:837-841. [PubMed: 10360120]

11. Mill J, Petronis A. Molecular studies of major depressive disorder: the epigenetic perspective. Mol Psychiatry 2007;12:799-814. [PubMed: 17420765]

12. Hasler G, Drevets WC, Manji HK, Charney DS. Discovering endophenotypes for major depression. Neuropsychopharmacology 2004;29:1765-1781. [PubMed: 15213704]

13. Ressler KJ, Mayberg HS. Targeting abnormal neural circuits in mood and anxiety disorders: from the laboratory to the clinic. Nature Neurosci 2007;10:1116-1124. [PubMed: 17726478]

14. Berton O, Nestler EJ. New approaches to antidepressant drug discovery: beyond monoamines. Nature Rev Neurosci 2006;7:137-151. [PubMed: 16429123]

15. Sheline YI. Neuroimaging studies of mood disorder effects on the brain. Biol Psychiatry 2003;54:338352. [PubMed: 12893109]

16. Harrison PJ. The neuropathology of primary mood disorder. Brain 2002;125:1428-1449. [PubMed: 12076995]

17. Mayberg HS, et al. Deep brain stimulation for treatment-resistant depression. Neuron 2005;45:651660. [PubMed: 15748841]This paper gives the first demonstration of the therapeutic efficacy of deep brain stimulation applied to the subgenual cingulate gyrus for treatment-refractory depression

18. Schlaepfer TE, et al. Deep brain stimulation to reward circuitry alleviates anhedonia in refractory major depression. Neuropsychopharmacology 2008;33:368-377. [PubMed: 17429407]

19. Nestler EJ, Carlezon WA Jr. The mesolimbic dopamine reward circuit in depression. Biol Psychiatry 2006;59:1151-1159. [PubMed: 16566899]

20. Pittenger C, Duman RS. Stress, depression, and neuroplasticity: a convergence of mechanisms. Neuropsychopharmacology 2008;33:88-109. [PubMed: 17851537]

21. Trivedi MH, et al. Evaluation of outcomes with citalopram for depression using measurement-based care in STAR*D: implications for clinical practice. Am J Psychiatry 2006;163:28-40. [PubMed: 16390886]

22. Ansorge MS, Hen R, Gingrich JA. Neurodevelopmental origins of depressive disorders. Curr Opin Pharmacol 2007;7:8-17. [PubMed: 17188022]

23. Ruhe HG, Mason NS, Schene AH. Mood is indirectly related to serotonin, norepinephrine and dopamine levels in humans: a meta-analysis of monoamine depletion studies. Mol Psychiatry 2007;12:331-359. [PubMed: 17389902]

24. $\mathrm{Hu} \mathrm{H}$, et al. Emotion enhances learning via norepinephrine regulation of AMPA-receptor trafficking. Cell 2007;131:160-173. [PubMed: 17923095]

25. Krishnan V, et al. Molecular adaptations underlying susceptibility and resistance to social defeat in brain reward regions. Cell 2007;131:391-404. [PubMed: 17956738]This paper adapts the socialdefeat model to study resilient behaviour and identifies active neurobiological mechanisms that maintain normal functioning in the face of chronic stress

26. Svenningsson P, et al. Alterations in 5-HT1B receptor function by 111 in depression-like states. Science 2006;311:77-80. [PubMed: 16400147]

27. Mathew SJ, Manji HK, Charney DS. Novel drugs and therapeutic targets for severe mood disorders. Neuropsychopharmacology 2008;33:2080-2092. [PubMed: 18172433]This paper provides an upto-date and comprehensive list of new antidepressant drugs currently in various stages of clinical trials 
28. Lucas G, et al. Serotonin 4 (5- $\left.\mathrm{HT}_{4}\right)$ receptor agonists are putative antidepressants with a rapid onset of action. Neuron 2007;55:712-725. [PubMed: 17785179]

29. Uhr M, et al. Polymorphisms in the drug transporter gene $A B C B 1$ predict antidepressant treatment response in depression. Neuron 2008;57:203-209. [PubMed: 18215618]

30. Holsboer F. How can we realize the promise of personalized antidepressant medicines? Nature Rev Neurosci 2008;9:638-646. [PubMed: 18628772]

31. Duman RS, Monteggia LM. A neurotrophic model for stress-related mood disorders. Biol Psychiatry 2006;59:1116-1127. [PubMed: 16631126]

32. Monteggia LM, et al. Essential role of brain-derived neurotrophic factor in adult hippocampal function. Proc Natl Acad Sci USA 2004;101:10827-10832. [PubMed: 15249684]

33. Karege F, Vaudan G, Schwald M, Perroud N, La Harpe R. Neurotrophin levels in postmortem brains of suicide victims and the effects of antemortem diagnosis and psychotropic drugs. Brain Res Mol Brain Res 2005;136:29-37. [PubMed: 15893584]

34. Shirayama Y, Chen AC, Nakagawa S, Russell DS, Duman RS. Brain-derived neurotrophic factor produces antidepressant effects in behavioral models of depression. J Neurosci 2002;22:3251-3261. [PubMed: 11943826]

35. Monteggia LM, et al. Brain-derived neurotrophic factor conditional knockouts show gender differences in depression-related behaviors. Biol Psychiatry 2007;61:187-197. [PubMed: 16697351]

36. Groves JO. Is it time to reassess the BDNF hypothesis of depression? Mol Psychiatry 2007;12:10791088. [PubMed: 17700574]

37. Martinowich K, Manji H, Lu B. New insights into BDNF function in depression and anxiety. Nature Neurosci 2007;10:1089-1093. [PubMed: 17726474]

38. Zorner B, et al. Forebrain-specific trkB-receptor knockout mice: behaviorally more hyperactive than 'depressive'. Biol Psychiatry 2003;54:972-982. [PubMed: 14625139]

39. Berton O, et al. Essential role of BDNF in the mesolimbic dopamine pathway in social defeat stress. Science 2006;311:864-868. [PubMed: 16469931]By using the social-defeat model, this paper characterizes the potent, pro-depressant effects of BDNF in the mesolimbic dopamine pathway, which is opposite to the well-described antidepressant-like effects of BDNF in hippocampal circuits

40. Eisch AJ, et al. Brain-derived neurotrophic factor in the ventral midbrain-nucleus accumbens pathway: a role in depression. Biol Psychiatry 2003;54:994-1005. [PubMed: 14625141]

41. Chen ZY, et al. Genetic variant BDNF (Val66Met) polymorphism alters anxiety-related behavior. Science 2006;314:140-143. [PubMed: 17023662]

42. Egan MF, et al. The BDNF Val66Met polymorphism affects activity-dependent secretion of BDNF and human memory and hippocampal function. Cell 2003;112:257-269. [PubMed: 12553913]This multidisciplinary study demonstrates how the polymorphism in BDNF in which methionine is substituted for valine at position 66 causes deficits in episodic memory, alters hippocampal activation and decreases activity-dependent BDNF secretion

43. Szeszko PR, et al. Brain-derived neurotrophic factor Val66Met polymorphism and volume of the hippocampal formation. Mol Psychiatry 2005;10:631-636. [PubMed: 15768049]

44. Gratacos M, et al. Brain-derived neurotrophic factor Val66Met and psychiatric disorders: metaanalysis of case-control studies confirm association to substance-related disorders, eating disorders, and schizophrenia. Biol Psychiatry 2007;61:911-922. [PubMed: 17217930]

45. Kaufman J, et al. Brain-derived neurotrophic factor-5-HTTLPR gene interactions and environmental modifiers of depression in children. Biol Psychiatry 2006;59:673-680. [PubMed: 16458264]

46. Kim JM, et al. Interactions between life stressors and susceptibility genes (5-HTTLPR and BDNF) on depression in Korean elders. Biol Psychiatry 2007;62:423-428. [PubMed: 17482146]

47. Pezawas L, et al. Evidence of biologic epistasis between BDNF and SLC6A4 and implications for depression. Mol Psychiatry 2008;13:709-716. [PubMed: 18347599]

48. Sahay A, Hen R. Adult hippocampal neurogenesis in depression. Nature Neurosci 2007;10:11101115. [PubMed: 17726477]

49. Sairanen M, Lucas G, Ernfors P, Castren M, Castren E. Brain-derived neurotrophic factor and antidepressant drugs have different but coordinated effects on neuronal turnover, proliferation, and survival in the adult dentate gyrus. J Neurosci 2005;25:1089-1094. [PubMed: 15689544] 
50. Hunsberger JG, et al. Antidepressant actions of the exercise-regulated gene VGF. Nature Med 2007;13:1476-1482. [PubMed: 18059283]This paper characterizes the antidepressant effects of VGF, an exercise-regulated neurotrophic factor in the hippocampus, and identifies VGF-mediated signalling as a potential therapeutic target

51. Thakker-Varia S, et al. The neuropeptide VGF produces antidepressant-like behavioral effects and enhances proliferation in the hippocampus. J Neurosci 2007;27:12156-12167. [PubMed: 17989282]

52. Warner-Schmidt JL, Duman RS. VEGF is an essential mediator of the neurogenic and behavioral actions of antidepressants. Proc Natl Acad Sci USA 2007;104:4647-4652. [PubMed: 17360578]

53. Airan RD, et al. High-speed imaging reveals neurophysiological links to behavior in an animal model of depression. Science 2007;317:819-823. [PubMed: 17615305] This study uses quantitative voltagesensitive dye imaging to explore the contribution of antidepressant-induced neurogenesis to local hippocampal network dynamics

54. Kempermann G. The neurogenic reserve hypothesis: what is adult hippocampal neurogenesis good for? Trends Neurosci 2008;31:163-169. [PubMed: 18329110]

55. Surget A, et al. Drug-dependent requirement of hippocampal neurogenesis in a model of depression and of antidepressant reversal. Biol Psychiatry 2008;64:293-301. [PubMed: 18406399]

56. Santarelli L, et al. Requirement of hippocampal neurogenesis for the behavioral effects of antidepressants. Science 2003;301:805-809. [PubMed: 12907793]

57. Zhao C, Deng W, Gage FH. Mechanisms and functional implications of adult neurogenesis. Cell 2008;132:645-660. [PubMed: 18295581]

58. Parker KJ, Schatzberg AF, Lyons DM. Neuroendocrine aspects of hypercortisolism in major depression. Horm Behav 2003;43:60-66. [PubMed: 12614635]

59. Raison CL, Miller AH. When not enough is too much: the role of insufficient glucocorticoid signaling in the pathophysiology of stress-related disorders. Am J Psychiatry 2003;160:1554-1565. [PubMed: 12944327]

60. Gourley SL, et al. Regionally specific regulation of ERK MAP kinase in a model of antidepressantsensitive chronic depression. Biol Psychiatry 2007;63:353-359. [PubMed: 17889834]

61. McEwen BS. Physiology and neurobiology of stress and adaptation: central role of the brain. Physiol Rev 2007;87:873-904. [PubMed: 17615391]

62. Brown ES, Varghese FP, McEwen BS. Association of depression with medical illness: does cortisol play a role? Biol Psychiatry 2004;55:1-9. [PubMed: 14706419]

63. Nemeroff CB, Owens MJ. Treatment of mood disorders. Nature Neurosci 2002;5 (Suppl):1068-1070. [PubMed: 12403988]

64. de Kloet ER, Joels M, Holsboer F. Stress and the brain: from adaptation to disease. Nature Rev Neurosci 2005;6:463-475. [PubMed: 15891777]

65. Brouwer JP, et al. Thyroid and adrenal axis in major depression: a controlled study in outpatients. Eur J Endocrinol 2005;152:185-191. [PubMed: 15745924]

66. Schatzberg AF, Lindley S. Glucocorticoid antagonists in neuropsychotic disorders. Eur J Pharmacol 2008;583:358-364. [PubMed: 18339372]

67. Gold PW, Chrousos GP. Organization of the stress system and its dysregulation in melancholic and atypical depression: high vs low CRH/NE states. Mol Psychiatry 2002;7:254-275. [PubMed: 11920153]

68. Heim C, Ehlert U, Hellhammer DH. The potential role of hypocortisolism in the pathophysiology of stress-related bodily disorders. Psychoneuroendocrinology 2000;25:1-35. [PubMed: 10633533]

69. Dantzer R, O’Connor JC, Freund GG, Johnson RW, Kelley KW. From inflammation to sickness and depression: when the immune system subjugates the brain. Nature Rev Neurosci 2008;9:46-56. [PubMed: 18073775]

70. Dunn AJ, Swiergiel AH, de Beaurepaire R. Cytokines as mediators of depression: what can we learn from animal studies? Neurosci Biobehav Rev 2005;29:891-909. [PubMed: 15885777]

71. Loftis JM, Hauser P. The phenomenology and treatment of interferon-induced depression. J Affect Disord 2004;82:175-190. [PubMed: 15488246]

72. Chourbaji S, et al. IL-6 knockout mice exhibit resistance to stress-induced development of depressionlike behaviors. Neurobiol Dis 2006;23:587-594. [PubMed: 16843000] 
73. Simen BB, Duman CH, Simen AA, Duman RS. TNF $\alpha$ signaling in depression and anxiety: behavioral consequences of individual receptor targeting. Biol Psychiatry 2006;59:775-785. [PubMed: 16458261]

74. Koo JW, Duman RS. IL-1 $\beta$ is an essential mediator of the antineurogenic and anhedonic effects of stress. Proc Natl Acad Sci USA 2008;105:751-756. [PubMed: 18178625]

75. Tsankova N, Renthal W, Kumar A, Nestler EJ. Epigenetic regulation in psychiatric disorders. Nature Rev Neurosci 2007;8:355-367. [PubMed: 17453016]

76. Szyf M, Weaver I, Meaney M. Maternal care, the epigenome and phenotypic differences in behavior. Reprod Toxicol 2007;24:9-19. [PubMed: 17561370]

77. Weaver IC, et al. Epigenetic programming by maternal behavior. Nature Neurosci 2004;7:847-854. [PubMed: 15220929]

78. Tsankova NM, Kumar A, Nestler EJ. Histone modifications at gene promoter regions in rat hippocampus after acute and chronic electroconvulsive seizures. J Neurosci 2004;24:5603-5610. [PubMed: 15201333]

79. Tsankova NM, et al. Sustained hippocampal chromatin regulation in a mouse model of depression and antidepressant action. Nature Neurosci 2006;9:519-525. [PubMed: 16501568]This paper contains one of the first demonstrations of the role of epigenetic modifications in stress-induced effects on the brain and their reversal by antidepressant treatments, as well as the therapeutic potential of HDAC inhibitors in depression

80. Schroeder FA, Lin CL, Crusio WE, Akbarian S. Antidepressant-like effects of the histone deacetylase inhibitor, sodium butyrate, in the mouse. Biol Psychiatry 2007;62:55-64. [PubMed: 16945350]

81. Renthal W, et al. Histone deacetylase 5 epigenetically controls behavioral adaptations to chronic emotional stimuli. Neuron 2007;56:517-529. [PubMed: 17988634]

82. Yehuda R. Risk and resilience in posttraumatic stress disorder. J Clin Psychiatry 2004;65 (Suppl 1): 29-36. [PubMed: 14728094]

83. Charney DS. Psychobiological mechanisms of resilience and vulnerability: implications for successful adaptation to extreme stress. Am J Psychiatry 2004;161:195-216. [PubMed: 14754765]

84. Berton $\mathrm{O}$, et al. Induction of $\triangle \mathrm{FosB}$ in the periaqueductal gray by stress promotes active coping responses. Neuron 2007;55:289-300. [PubMed: 17640529]In this paper, the learned-helplessness model is used to illustrate the pro-resilient effects of the transcription factor $\triangle F O S B$ within the dorsal raphe nucleus

85. Strekalova T, Spanagel R, Bartsch D, Henn FA, Gass P. Stress-induced anhedonia in mice is associated with deficits in forced swimming and exploration. Neuropsychopharmacology 2004;29:2007-2017. [PubMed: 15266352]

86. Sajdyk TJ, et al. Neuropeptide $\mathrm{Y}$ in the amygdala induces long-term resilience to stress-induced reductions in social responses but not hypothalamic-adrenal-pituitary axis activity or hyperthermia. J Neurosci 2008;28:893-903. [PubMed: 18216197]

87. Peaston AE, Whitelaw E. Epigenetics and phenotypic variation in mammals. Mamm Genome 2006;17:365-374. [PubMed: 16688527]

88. Bergstrom A, Jayatissa MN, Thykjaer T, Wiborg O. Molecular pathways associated with stress resilience and drug resistance in the chronic mild stress rat model of depression: a gene expression study. J Mol Neurosci 2007;33:201-215. [PubMed: 17917079]

89. Zarate CA Jr, et al. A randomized trial of an N-methyl-D-aspartate antagonist in treatment-resistant major depression. Arch Gen Psychiatry 2006;63:856-864. [PubMed: 16894061]

90. Maeng S, et al. Cellular mechanisms underlying the antidepressant effects of ketamine: role of $\alpha$ amino-3-hydroxy-5-methylisoxazole-4-propionic acid receptors. Biol Psychiatry 2008;63:349-352. [PubMed: 17643398]

91. Garcia LS, et al. Acute administration of ketamine induces antidepressant-like effects in the forced swimming test and increases BDNF levels in the rat hippocampus. Prog Neuropsychopharmacol Biol Psychiatry 2008;32:140-144. [PubMed: 17884272]

92. Maeng S, Zarate CA Jr. The role of glutamate in mood disorders: results from the ketamine in major depression study and the presumed cellular mechanism underlying its antidepressant effects. Curr Psychiatry Rep 2007;9:467-474. [PubMed: 18221626] 
93. Roy M, David N, Cueva M, Giorgetti M. A study of the involvement of melanin-concentrating hormone receptor 1 (MCHR1) in murine models of depression. Biol Psychiatry 2007;61:174-180. [PubMed: 16934771]

94. Georgescu D, et al. The hypothalamic neuropeptide melanin-concentrating hormone acts in the nucleus accumbens to modulate feeding behavior and forced-swim performance. J Neurosci 2005;25:2933-2940. [PubMed: 15772353]

95. Lutter M, et al. The orexigenic hormone ghrelin defends against depressive symptoms of chronic stress. Nature Neurosci 2008;11:752-753. [PubMed: 18552842]

96. Lu XY, Kim CS, Frazer A, Zhang W. Leptin: a potential novel antidepressant. Proc Natl Acad Sci USA 2006;103:1593-1598. [PubMed: 16423896]

97. Kishi T, Elmquist JK. Body weight is regulated by the brain: a link between feeding and emotion. Mol Psychiatry 2005;10:132-146. [PubMed: 15630408]

98. Kaplitt MG, et al. Safety and tolerability of gene therapy with an adeno-associated virus (AAV) borne GAD gene for Parkinson's disease: an open label, phase I trial. Lancet 2007;369:2097-2105.

[PubMed: 17586305]This paper demonstrates the efficacy and safety of stereotactic viral-mediated gene therapy in the treatment of severe Parkinson's disease

99. Cryan JF, Markou A, Lucki I. Assessing antidepressant activity in rodents: recent developments and future needs. Trends Pharmacol Sci 2002;23:238-245. [PubMed: 12008002] 


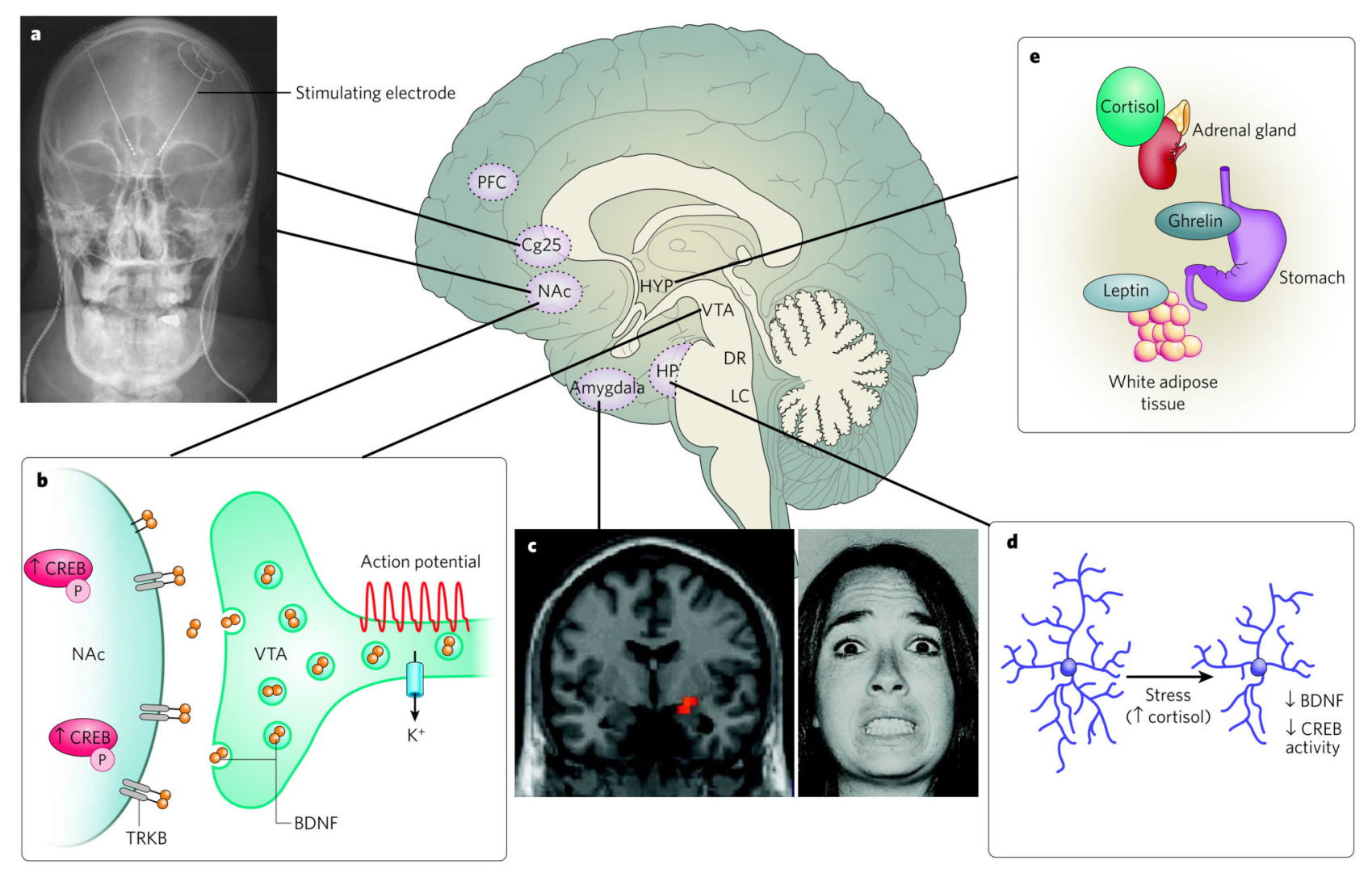

Figure 1. Neural circuitry of depression

Several brain regions are implicated in the pathophysiology of depression. a, Deep brain stimulation of the subgenual cingulate cortex $(\mathrm{Cg} 25)^{17}$ or the nucleus accumbens (NAc) ${ }^{18}$ has an antidepressant effect on individuals who have treatment-resistant depression. This effect is thought to be mediated through inhibiting the activity of these regions either by depolarization blockade or by stimulation of passing axonal fibres. (Image courtesy of T. Schlaepfer and V. Sturm, University Hospital, Bonn, Germany.) b, Increased activity-dependent release of brainderived neurotrophic factor (BDNF) within the mesolimbic dopamine circuit (dopamineproducing ventral tegmental area (VTA) to dopamine-sensitive NAc) mediates susceptibility to social stress ${ }^{25}$, probably occurring in part through activation of the transcription factor CREB (cyclic-AMP-response-element-binding protein) ${ }^{20}$ by phosphorylation $(\mathrm{P})$. c, Neuroimaging studies strongly implicate the amygdala (red pixels show activated areas) as an important limbic node for processing emotionally salient stimuli, such as fearful faces ${ }^{7}$. (Image courtesy of D. Weinberger, National Institute of Mental Health, Bethesda, Maryland). d, Stress decreases the concentrations of neurotrophins (such as BDNF), the extent of neurogenesis and the complexity of neuronal processes in the hippocampus (HP), effects that are mediated in part through increased cortisol concentrations and decreased CREB activity ${ }^{2,14}$. e, Peripherally released metabolic hormones in addition to cortisol, such as ghrelin ${ }^{95}$ and leptin 96 , produce moodrelated changes through their effects on the hypothalamus (HYP) and several limbic regions (for example, the hippocampus, VTA and NAc). DR, dorsal raphe; LC, locus coeruleus; PFC, prefrontal cortex. 
a
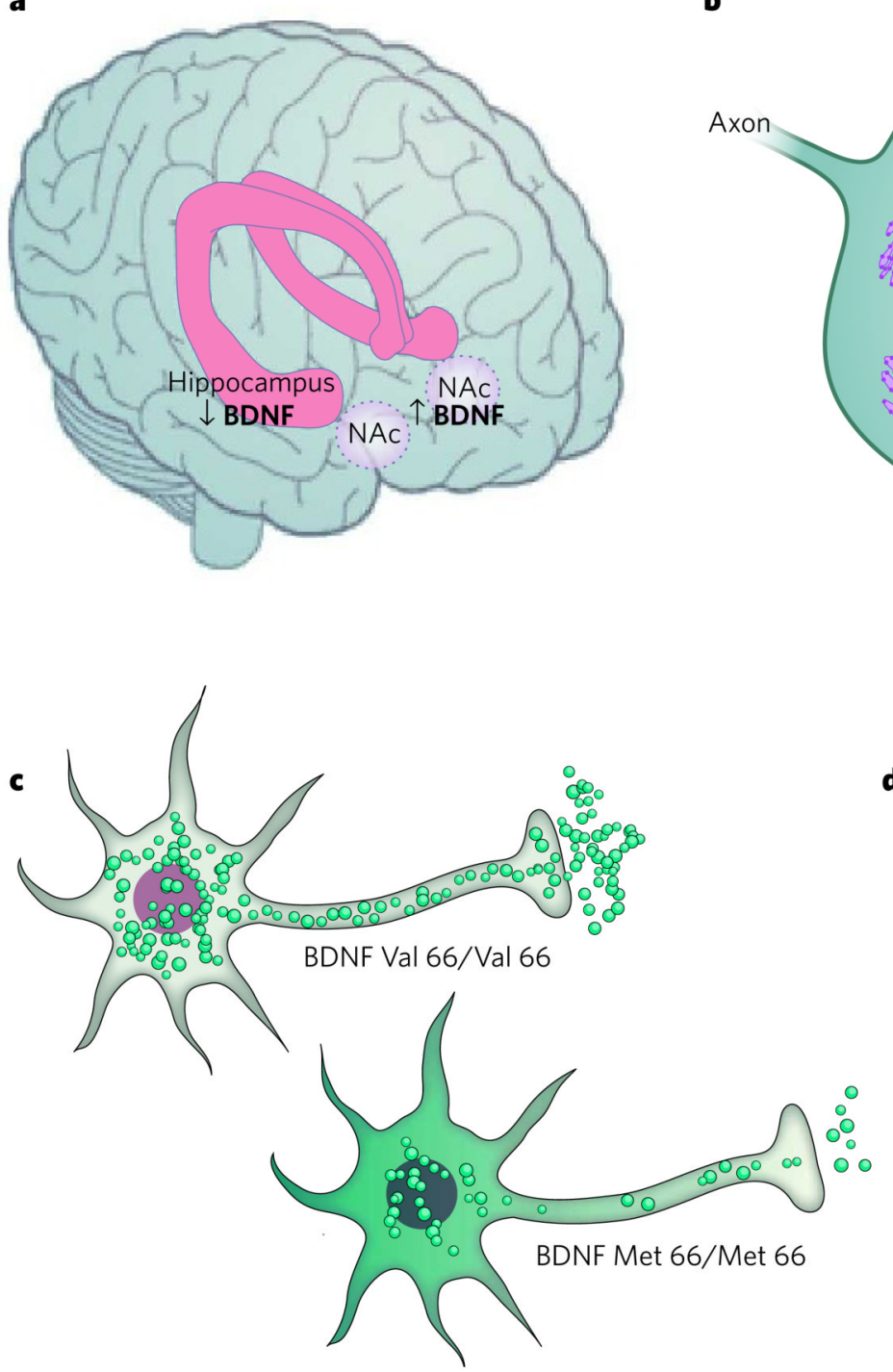

b

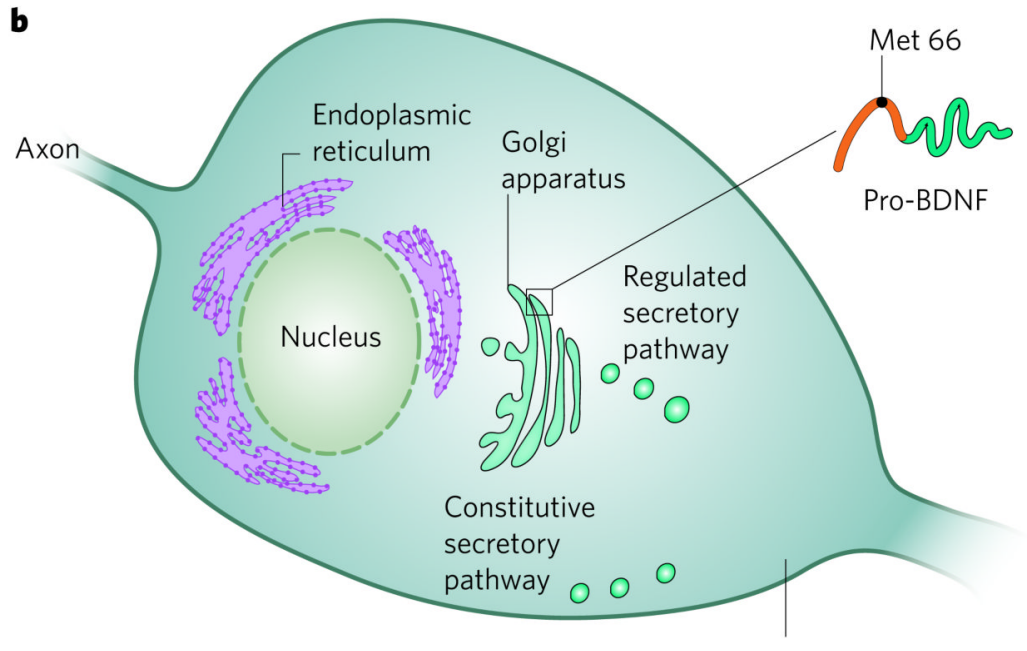

Neuron cell body

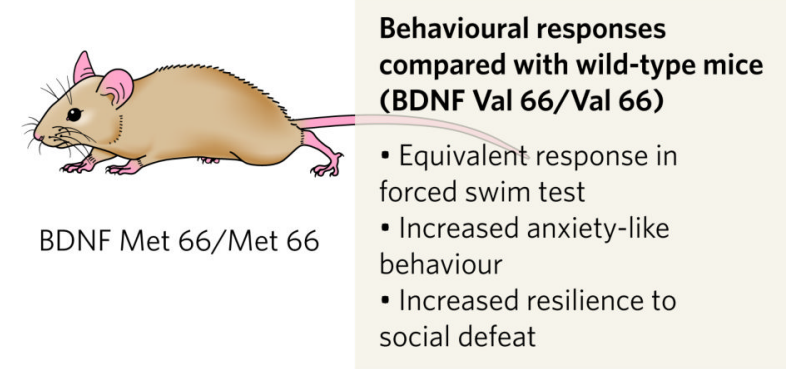

Figure 2. BDNF and depression - an example of the complexities of the molecular pathophysiology of depression

a, Post-mortem data from depressed humans show that depression is associated with a decrease in the amount of BDNF in the hippocampus ${ }^{33}$ and an increase (of similar magnitude) in the $\mathrm{NAc}^{25}$, an example of the regional specificity of depression-related neuroplastic changes. $\mathbf{b}$, Neuronal secretion of BDNF occurs through regulated (activity-dependent) and constitutive secretory pathways. Regulated secretion is modulated by the interactions of proteins in the Golgi apparatus with the pro-domain of BDNF, the site of a single-nucleotide polymorphism (G196A) in humans that results in the substitution of valine at amino-acid residue 66 with methionine. c, The Met-66-containing BDNF variant has impaired intracellular trafficking. Met-66 BDNF is not properly sorted within the cell, causing it to be distributed throughout the cell body outside of vesicles ${ }^{42}$. In addition, less BDNF is secreted from the nerve terminal. d, Knock-in mice that homozygously express Met-66 BDNF ${ }^{41}$ have normal responses in the forced-swim test ${ }^{25}$, but these mice show more anxiety-like behaviour ${ }^{41}$ and greater resilience to behavioural and molecular changes after social defeat ${ }^{25}$, implicating this BDNF polymorphism in the pathophysiology of psychological disorders that are influenced by stressful life events. 
a Histone methylation (repressive)

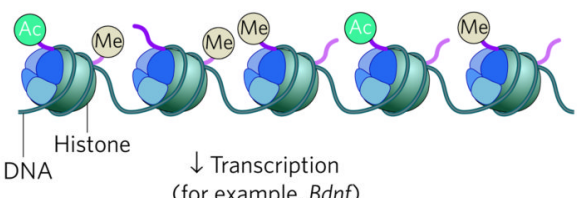

(for example, Bdnf)

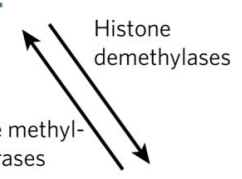

\begin{tabular}{c}
$\begin{array}{c}\text { Early life } \\
\text { stress }\end{array}$ \\
\hline
\end{tabular}

DNA methyl-

transferases b DNA methylation (repressive)

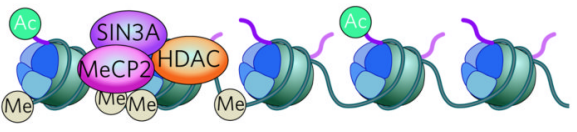

$\downarrow$ Transcription

(for example, glucocorticoid receptor gene)

Figure 3. Epigenetic regulation in depression

The transcriptional potential of genes involved in neuroplastic responses to stress or antidepressant treatments can be regulated through chromatin-remodelling events catalysed by specific enzymes. a, The methylation of histones on specific lysine residues (for example, Lys 9 and Lys 27) is associated with condensed chromatin (heterochromatin) and is important in the repression of $B d n f$ expression in the hippocampus after social defeat ${ }^{79}$. The pluses and minuses indicate activation or inhibition, respectively, of a particular process. b. By contrast, repression of other genes can occur through the methylation of cytosine within $\mathrm{CpG}$ islands of promoter regions, attracting proteins involved in transcriptional repression, such as SIN3A, $\mathrm{MeCP} 2$ (methyl-CpG binding protein 2) and histone deacetylases (HDACs). DNA methylation of the promoter of the glucocorticoid receptor gene occurs in rat pups born to mothers with inherently low levels of maternal behaviour ${ }^{77}$. Although such methylation events have been reported to be reversible, the enzymes responsible for demethylating DNA have yet to be identified ${ }^{75,76}$. c, Histone acetylation, catalysed by histone acetyltransferases, is associated with decondensed chromatin (euchromatin), increasing the activity of transcriptional complexes. HDAC inhibitors (which activate the expression of numerous genes that have not yet been identified with certainty) show antidepressant properties in several assays ${ }^{79,80}$. Ac, acetyl; Me, methyl. 
Table 1

A systematic comparison of major depression and type 2 diabetes

\begin{tabular}{|c|c|c|}
\hline Criterion & Major depressive disorder & Type 2 diabetes \\
\hline Lifetime risk & 1 in 6 & 1 in 3 \\
\hline \multirow[t]{2}{*}{ Diagnosis and monitoring } & $\begin{array}{l}\text { Subjective-qualitative: patients must show a depressed mood } \\
\text { or anhedonia, as well as assorted other symptoms, for at least } \\
2 \text { weeks, and these symptoms must disrupt normal social and } \\
\text { occupational functioning }\end{array}$ & $\begin{array}{l}\text { Objective-quantitative: diagnosis } \\
\text { requires demonstration of an increased } \\
\text { amount of serum glucose with classical } \\
\text { signs (polyuria, polydipsia, obesity) or } \\
\text { abnormal glucose tolerance (reflecting } \\
\text { insulin resistance) }\end{array}$ \\
\hline & Patients monitored through standardized questionnaires & $\begin{array}{l}\text { Significant increases in } \mathrm{HbA} 1 \mathrm{C}, \text { a } \\
\text { glycosylated haemoglobin, demonstrate } \\
\text { long-standing poor glycaemic control }\end{array}$ \\
\hline \multirow[t]{3}{*}{ Aetiology and risk factors } & $\begin{array}{l}\text { Stressful life events (such as loss of loved ones or financial or } \\
\text { professional crises) }\end{array}$ & $\begin{array}{l}\text { Lifestyle factors (sedentary lifestyle, } \\
\text { high-fat diet) }\end{array}$ \\
\hline & Genetic risk (heritability $\approx 40 \%$ ) & Genetic risk (heritability $\approx 35 \%$ ) \\
\hline & $\begin{array}{l}\text { Disease genes unknown; can be idiopathic, a side effect of a } \\
\text { drug (such as interferon- } \alpha \text { or isotretinoin) or secondary to } \\
\text { systemic illness (such as Cushing's syndrome or stroke, } \\
\text { among many others) }\end{array}$ & $\begin{array}{l}\text { Established disease genes (such as } \\
P P A R G, T C F 7 L 2 \text { or } K C N J 11) \text {; can be } \\
\text { iatrogenic (such as treatment with } \\
\text { glucocorticoids or phenytoin) }\end{array}$ \\
\hline \multirow[t]{8}{*}{ Treatments } & $\begin{array}{l}\text { Monoamine reuptake inhibitors (such as tricyclic drugs, } \\
\text { SSRIs, NRIs or SNRIs) }\end{array}$ & Insulin \\
\hline & Monoamine oxidase inhibitors (such as tranylcypromine) & Sulphonylureas (such as tolbutamide) \\
\hline & 'Atypical' agents (such as bupropion or mirtazapine) & Meglitinides (such as repaglinide) \\
\hline & Electroconvulsive seizures & PPAR- $\gamma$ agonists (such as rosiglitazone) \\
\hline & Psychotherapy & Biguanides (such as metformin) \\
\hline & Deep brain stimulation & Glucosidase inhibitors (such as miglitol) \\
\hline & Exercise promotes recovery & $\begin{array}{l}\text { Incretin (GLP1) mimetics (such as } \\
\text { exenatide) }\end{array}$ \\
\hline & & $\begin{array}{l}\text { Lifestyle changes (such as weight loss or } \\
\text { exercise) }\end{array}$ \\
\hline
\end{tabular}

Pathogenesis Abnormal activity of the HPA axis (hypercortisolism or
hypocortisolism)?

Alterations in neurotrophic signalling?

Abnormal hippocampal neurogenesis?

Deficits in brain reward processing?

Abnormal cognitive styles (negative thinking)?
Obesity, sedentariness and genetic predisposition promote peripheral insulin resistance leading to pancreatic $\beta$-cell hyperplasia

$\beta$-Cell dysfunction and failure ensues, leading to impaired glucose tolerance

End-organ damage (nephropathy, neuropathy and angiopathy) occurs secondarily to hyperglycaemia, excessive protein glycation and aberrant intracellular signalling

GLP1, glucagon-like peptide 1; HbA1C, haemoglobin A1 C; HPA, hypothalamic-pituitary-adrenal axis; $K C N J 11$, potassium inwardly rectifying channel J11 gene; NRIs, selective noradrenaline reuptake inhibitors; $P P A R G$, peroxisome-proliferator-activated receptor- $\gamma$ gene; SNRI, serotonin-noradrenaline reuptake inhibitor; $T C F 7 L 2$, transcription factor 7 like 2 gene. 